\title{
Painlevé VI and Hankel determinants for the generalized Jacobi weight
}

\author{
D. Dai * \\ Department of Mathematics, City University of Hong Kong, \\ Tat Chee Avenue, Kowloon, Hong Kong \\ dandai@cityu.edu.hk \\ and \\ L. Zhang ${ }^{\dagger}$ \\ Department of Mathematics, Katholieke Universiteit Leuven, \\ Celestijnenlaan 200 B, 3001 Leuven, Belgium \\ lun.zhang@wis.kuleuven.be
}

October 27, 2018

\begin{abstract}
We study the Hankel determinant of the generalized Jacobi weight $(x-$ $t)^{\gamma} x^{\alpha}(1-x)^{\beta}$ for $x \in[0,1]$ with $\alpha, \beta>0, t<0$ and $\gamma \in \mathbb{R}$. Based on the ladder operators for the corresponding monic orthogonal polynomials $P_{n}(x)$, it is shown that the logarithmic derivative of Hankel determinant is characterized by a $\tau$-function for the Painlevé VI system.
\end{abstract}

*The work of this author was partially supported by the General Research Fund of Hong Kong (Project No. 9041431)

${ }^{\dagger}$ Corresponding author 


\section{Introduction and statement of results}

Let $P_{n}(x)$ be the monic polynomials of degree $n$ in $x$ and orthogonal with respect to the generalized Jacobi weight $w(x ; t)$; that is

$$
\int_{0}^{1} P_{m}(x) P_{n}(x) w(x) d x=h_{n} \delta_{m, n}, \quad h_{n}>0, \quad m, n=0,1,2, \cdots,
$$

where

$$
P_{n}(x)=x^{n}+\mathrm{p}_{1}(n) x^{n-1}+\cdots
$$

and

$$
w(x):=w(x ; t)=(x-t)^{\gamma} x^{\alpha}(1-x)^{\beta}, \quad x \in[0,1],
$$

with $\alpha, \beta>0, t<0$ and $\gamma \in \mathbb{R}$. (In what follows, we often suppress the $t$-dependence for brevity. We believe that this will not lead to any confusion.) An immediate consequence of the orthogonality condition is the three-term recurrence relation

$$
x P_{n}(x)=P_{n+1}(x)+\alpha_{n} P_{n}(x)+\beta_{n} P_{n-1}(x),
$$

where the "initial" conditions are taken to be $P_{0}(x):=1$ and $\beta_{0} P_{-1}(x):=0$. Obviously, when $t \rightarrow 0^{-}$, these polynomials are reduced to the classical Jacobi polynomials up to some shift and rescaling, whose properties are well-known; see Szegő [24]. In the literature, although people may use slightly different definitions, the generalized Jacobi polynomials have been studied from many points of view; for example, see [14, 15, 21, 25, 26]. In particular, Magnus [19] showed that an auxiliary quantity occurring in his study satisfies the Painlevé VI equation for certain parameters.

In this paper, we are concerned with the Hankel determinant for the generalized Jacobi weight

$$
\begin{aligned}
D_{n}(t) & =\operatorname{det}\left(\int_{0}^{1} x^{j+k} w(x ; t) d t\right)_{j, k=0}^{n-1} \\
& =\frac{1}{n !} \int_{0}^{1} \cdots \int_{0}^{1} \prod_{i<j}\left(x_{i}-x_{j}\right)^{2} \prod_{k=1}^{n} w\left(x_{k} ; t\right) d x_{k}
\end{aligned}
$$

with $w(x ; t)$ given in (1.3). Our motivation for this research mainly arises from the close relation between Hankel determinants and random matrix theory, which is of interest in mathematical physics. Indeed, the Hankel determinant defined in (1.5) can be viewed as the partition function for the unitary ensemble with eigenvalue distribution

$$
\prod_{i<j}\left(x_{i}-x_{j}\right)^{2} \prod_{k=1}^{n}\left(x_{k}-t\right)^{\gamma} x_{k}^{\alpha}\left(1-x_{k}\right)^{\beta} d x_{k}
$$


see the definitive book of Mehta [20] for a discussion of this topic. The main purpose of this work is to study the properties of $D_{n}(t)$ as a function of $t$. More precisely, we are going to show the logarithmic derivative of Hankel determinant $D_{n}(t)$ is characterized by a $\tau$-function for the Painlevé VI system. The appearance of Painlevé VI may not be so surprising somehow. As a matter of fact, it has been already known that, for some special weight functions, the corresponding Hankel determinants are connected to the well-known nonlinear ordinary differential equations - Painlevé equations. In particular, it is first shown in [16] that a gap probability in the Jacobi polynomial ensemble is related to Painlevé VI, see also [7, 13] for further discussion. For the present general Jacobi case, although it is natural to "guess" the existence of such link, the precise form and specific quantity related to, however, is not clear.

Our approach is based on the ladder operator for orthogonal polynomials, which has been successfully applied to many other polynomials before; see [2, 3, 8, 10, 11, 12]. The main result is the following.

Theorem 1.1. Let $H_{n}$ be the logarithmic derivative of the Hankel determinant with respect to $t$,

$$
H_{n}(t):=t(t-1) \frac{d}{d t} \ln D_{n}(t)
$$

and denote by

$$
\widetilde{H}_{n}:=H_{n}+d_{1} t+d_{2}
$$

with

$$
\begin{aligned}
& d_{1}=-n(n+\alpha+\beta+\gamma)-\frac{(\alpha+\beta)^{2}}{4} \\
& d_{2}=\frac{1}{4}[2 n(n+\alpha+\beta+\gamma)+\beta(\alpha+\beta)-\gamma(\alpha-\beta)]
\end{aligned}
$$

Then $\widetilde{H}_{n}$ satisfies the following Jimbo-Miwa-Okamoto $\sigma$-form of Painlevé VI in [18, 22]

$$
\begin{aligned}
& \widetilde{H}_{n}^{\prime}\left(t(t-1) \widetilde{H}_{n}^{\prime \prime}\right)^{2}+\left\{2 \widetilde{H}_{n}^{\prime}\left(t \widetilde{H}_{n}^{\prime}-\widetilde{H}_{n}\right)-\widetilde{H}_{n}^{\prime 2}-\nu_{1} \nu_{2} \nu_{3} \nu_{4}\right\}^{2} \\
& =\left(\widetilde{H}_{n}^{\prime}+\nu_{1}^{2}\right)\left(\widetilde{H}_{n}^{\prime}+\nu_{2}^{2}\right)\left(\widetilde{H}_{n}^{\prime}+\nu_{3}^{2}\right)\left(\widetilde{H}_{n}^{\prime}+\nu_{4}^{2}\right)
\end{aligned}
$$

with

$$
\nu_{1}=\frac{\alpha+\beta}{2}, \quad \nu_{2}=\frac{\beta-\alpha}{2}, \quad \nu_{3}=\frac{2 n+\alpha+\beta}{2}, \quad \nu_{4}=\frac{2 n+\alpha+\beta+2 \gamma}{2} .
$$


Remark 1.1. Due to the symmetric form of (1.9), the choice of $\nu_{1}, \nu_{2}, \nu_{3}$ and $\nu_{4}$ is not unique.

Remark 1.2. Although we assume $t<0$ in the definition (1.3) of the weight function $w(x)$, Theorem 1.1 also holds for $t>1$ if $(x-t)^{\gamma}$ is substituted by $(t-x)^{\gamma}$. One may expect this theorem is valid for all real $t \neq 0,1$ when $(x-t)^{\gamma}$ is replaced by $|x-t|^{\gamma}$, which is similar to what has been studied by Chen and Feigin [9]. Unfortunately, we can not prove it at this moment.

Remark 1.3. If $\gamma=0$, then $(x-t)^{\gamma} \equiv 1$ and we can readily reduce the polynomials $P_{n}(x)$ in (1.1) to the classical Jacobi polynomials, which are of course $t$ independent. As a consequence, the formula (1.8) provides a trivial solution for the associated $\sigma$-form in (1.9). Moreover, if $\gamma=1$, the Hankel determinant $D_{n}(t)$ defined in (1.5) is actually a polynomial in $t$ of degree $n$, and orthogonal with respect to the "shifted" Jacobi weight $t^{\alpha}(1-t)^{\beta}$ on $[0,1]$. By the classical theory of Jacobi polynomials (cf. [24]), $D_{n}(t)$ satisfies the following second-order differential equation

$$
t(1-t) D_{n}^{\prime \prime}-[(2+\alpha+\beta) t-\alpha-1] D_{n}^{\prime}+n(n+\alpha+\beta+1) D_{n}=0 .
$$

Hence, if we denote by $u(t):=\frac{d}{d t} \ln D_{n}(t)$, it is easily seen that $u(t)$ is a solution of the following Riccati equation

$$
t(1-t) u^{\prime}=t(t-1) u^{2}+[(2+\alpha+\beta) t-\alpha-1] u-n(n+\alpha+\beta+1)
$$

for $t<0$ in this special case. Moreover, one can verify that $t(t-1) u(t)+d_{1} t+d_{2}$ is a rational solution to the associated $\sigma$-form in (1.9).

From (1.4), it is easily seen that $\beta_{n}=h_{n} / h_{n-1}$. Since

$$
D_{n}(t)=\prod_{j=0}^{n-1} h_{j}
$$

(see Eq.(2.1.6) in [17]), we can express $\beta_{n}$ in terms of the Hankel determinant as follows

$$
\beta_{n}=\frac{D_{n-1} D_{n+1}}{D_{n}^{2}} .
$$

Therefore, it is also expected that there exists certain relation between $\beta_{n}$ and the Painlevé VI equation. Indeed, as a by-product of our main theorem, we find a firstorder differential equation for $\beta_{n}$, whose coefficient is closely related to the Painlevé VI equation. 
Theorem 1.2. The recurrence coefficient $\beta_{n}$ satisfies a first-order differential equation as follows

$$
t \frac{d}{d t} \beta_{n}=\left(2+R_{n-1}-R_{n}\right) \beta_{n},
$$

where $R_{n}$ is related to the Painleve VI equation in the following way. Let

$$
W_{n}(t):=\frac{(t-1) R_{n}(t)}{2 n+\alpha+\beta+\gamma+1}+1 .
$$

Then $W_{n}(t)$ satisfies the Painlevé VI equation

$$
\begin{aligned}
W_{n}^{\prime \prime}= & \frac{1}{2}\left(\frac{1}{W_{n}}+\frac{1}{W_{n}-1}+\frac{1}{W_{n}-t}\right)\left(W_{n}^{\prime}\right)^{2}-\left(\frac{1}{t}+\frac{1}{t-1}+\frac{1}{W_{n}-t}\right) W_{n}^{\prime} \\
& +\frac{W_{n}\left(W_{n}-1\right)\left(W_{n}-t\right)}{t^{2}(t-1)^{2}}\left(\mu_{1}+\frac{\mu_{2} t}{W_{n}^{2}}+\frac{\mu_{3}(t-1)}{\left(W_{n}-1\right)^{2}}+\frac{\mu_{4} t(t-1)}{\left(W_{n}-t\right)^{2}}\right)
\end{aligned}
$$

with

$$
\mu_{1}=\frac{(2 n+\alpha+\beta+\gamma+1)^{2}}{2}, \quad \mu_{2}=-\frac{\alpha^{2}}{2}, \quad \mu_{3}=\frac{\beta^{2}}{2}, \quad \mu_{4}=\frac{1-\gamma^{2}}{2} .
$$

The present paper is organized as follows. In Section 2, we give a brief introduction to the ladder operator theory and state three compatibility conditions $S_{1}, S_{2}$ and $S_{2}^{\prime}$. Based on these supplementary conditions, we introduce some auxiliary constants in Section 3, Their relations with other quantities such as the coefficient of orthogonal polynomials, Hankel determinant, etc., are also derived for further use. We conclude this paper with the proof of Theorem 1.1 and Theorem 1.2 in Sections 4 and 5 , respectively.

\section{Ladder operators and compatibility conditions}

The ladder operators for orthogonal polynomials has been derived by many authors with a long history, we refer to [4, 5, 6, 11, 23] and references therein for a quick guide. Following the general set-up (see [11] for example), we have the lowering and raising ladder operator for our generalized Jacobi polynomials $P_{n}(z)$ :

$$
\begin{aligned}
\left(\frac{d}{d z}+B_{n}(z)\right) P_{n}(z) & =\beta_{n} A_{n}(z) P_{n-1}(z), \\
\left(\frac{d}{d z}-B_{n}(z)-\mathrm{v}^{\prime}(z)\right) P_{n-1}(z) & =-A_{n-1}(z) P_{n}(z)
\end{aligned}
$$


with $\vee(z):=-\ln w(z)$ and

$$
\begin{aligned}
& A_{n}(z):=\frac{1}{h_{n}} \int_{0}^{1} \frac{\mathrm{v}^{\prime}(z)-\mathrm{v}^{\prime}(y)}{z-y}\left[P_{n}(y)\right]^{2} w(y) d y \\
& B_{n}(z):=\frac{1}{h_{n-1}} \int_{0}^{1} \frac{\mathrm{v}^{\prime}(z)-\mathrm{v}^{\prime}(y)}{z-y} P_{n-1}(y) P_{n}(y) w(y) d y .
\end{aligned}
$$

Note that, $A_{n}(z)$ and $B_{n}(z)$ are not independent but satisfy the following supplementary conditions.

Proposition 2.1. The functions $A_{n}(z)$ and $B_{n}(z)$ defined in (2.3) and 2.4) satisfy the following compatibility conditions,

$$
\begin{gathered}
B_{n+1}(z)+B_{n}(z)=\left(z-\alpha_{n}\right) A_{n}(z)-\vee(z) \\
1+\left(z-\alpha_{n}\right)\left[B_{n+1}(z)-B_{n}(z)\right]=\beta_{n+1} A_{n+1}(z)-\beta_{n} A_{n-1}(z) .
\end{gathered}
$$

Proof. Using the recurrence relation and the Christoffel-Darboux formulas, all the formulas (2.1), (2.2), $\left(S_{1}\right)$ and $\left(S_{2}\right)$ can be derived by direct calculations. We refer to [4, 5, 6, 23] for details. Also, see [10, 11] for a recent proof.

From $\left(S_{1}\right)$ and $\left(S_{2}\right)$, we can derive another identity involving $\sum_{j=0}^{n-1} A_{j}$ which is very helpful in our subsequent analysis. We state the result in the following Proposition.

\section{Proposition 2.2.}

$$
B_{n}^{2}(z)+\vee(z) B_{n}(z)+\sum_{j=0}^{n-1} A_{j}(z)=\beta_{n} A_{n}(z) A_{n-1}(z) .
$$

Proof. See the proof of Theorem 2.2 in [8].

The conditions $S_{1}, S_{2}$ and $S_{2}^{\prime}$ are usually called the compatibility conditions for the ladder operators, which will play an important role in our future analysis. Although the author obtained an equivalent form of $S_{2}^{\prime}$ in [19], he did not further study it. We would also like to emphasize that, as in [8], the condition $S_{2}$ is essential in the present case (see Remark 3.1 below), while in [2] and [3], only the conditions $S_{1}$ and $S_{2}^{\prime}$ are sufficient to derive all the relations. 


\section{The analysis of the ladder operators}

\subsection{Some auxiliary constants}

To prove our results stated in Section 1, we would like to introduce some auxiliary constants first. For the weight function $w(z)$ given in (1.3), we know

$$
\mathrm{v}(z):=-\ln w(z)=-\alpha \ln z-\beta \ln (1-z)-\gamma \ln (z-t)
$$

Hence,

$$
\mathrm{v}^{\prime}(z)=-\frac{\alpha}{z}-\frac{\beta}{z-1}-\frac{\gamma}{z-t}
$$

and

$$
\frac{\mathbf{v}^{\prime}(z)-\mathbf{v}^{\prime}(y)}{z-y}=\frac{\alpha}{z y}+\frac{\beta}{(z-1)(y-1)}+\frac{\gamma}{(z-t)(y-t)} .
$$

Since the right-hand side of the above formula is rational in $z$, it is easily seen that both $A_{n}(z)$ and $B_{n}(z)$ are also rational in $z$ from their definitions in (2.3) and (2.4). More precisely, we have the following lemma.

Lemma 3.1. We have

$$
\begin{aligned}
& A_{n}(z)=\frac{R_{n}^{*}}{z}-\frac{R_{n}}{z-1}+\frac{R_{n}-R_{n}^{*}}{z-t} \\
& B_{n}(z)=\frac{r_{n}^{*}}{z}-\frac{r_{n}}{z-1}+\frac{r_{n}-r_{n}^{*}-n}{z-t}
\end{aligned}
$$

where

$$
\begin{aligned}
R_{n}^{*} & :=\frac{\alpha}{h_{n}} \int_{0}^{1}\left[P_{n}(y)\right]^{2} w(y) \frac{d y}{y} \\
R_{n} & :=\frac{\beta}{h_{n}} \int_{0}^{1}\left[P_{n}(y)\right]^{2} w(y) \frac{d y}{1-y} \\
r_{n}^{*} & :=\frac{\alpha}{h_{n-1}} \int_{0}^{1} P_{n-1}(y) P_{n}(y) w(y) \frac{d y}{y} \\
r_{n} & :=\frac{\beta}{h_{n-1}} \int_{0}^{1} P_{n-1}(y) P_{n}(y) w(y) \frac{d y}{1-y} .
\end{aligned}
$$

Proof. Inserting (3.3) into (2.3) gives us

$$
\begin{aligned}
A_{n}(z)=\frac{1}{h_{n}} & {\left[\frac{\alpha}{z} \int_{0}^{1}\left[P_{n}(y)\right]^{2} w(y) \frac{d y}{y}+\frac{\beta}{z-1} \int_{0}^{1}\left[P_{n}(y)\right]^{2} w(y) \frac{d y}{y-1}\right.} \\
+ & \left.\frac{\gamma}{z-t} \int_{0}^{1}\left[P_{n}(y)\right]^{2} w(y) \frac{d y}{y-t}\right] .
\end{aligned}
$$


Applying integration by parts, we obtain

$$
\int_{0}^{1}\left[P_{n}(y)\right]^{2} w(y) \mathfrak{v}^{\prime}(y) d y=-\int_{0}^{1}\left[P_{n}(y)\right]^{2} d w(y)=\int_{0}^{1} 2 P_{n}^{\prime}(y) P_{n}(y) w(y) d y=0 .
$$

On account of (3.2) and the above formula, we have

$$
\gamma \int_{0}^{1}\left[P_{n}(y)\right]^{2} w(y) \frac{d y}{y-t}=\beta \int_{0}^{1}\left[P_{n}(y)\right]^{2} w(y) \frac{d y}{1-y}-\alpha \int_{0}^{1}\left[P_{n}(y)\right]^{2} w(y) \frac{d y}{y} .
$$

A combination of (3.10) and (3.12) yields (3.4).

In a similar manner, we get (3.5) from (2.4). In that case, we need to make use of the following equality

$$
\begin{aligned}
\gamma \int_{0}^{1} P_{n-1}(y) P_{n}(y) w(y) \frac{d y}{y-t}= & -n h_{n-1}-\alpha \int_{0}^{1} P_{n-1}(y) P_{n}(y) w(y) \frac{d y}{y} \\
& +\beta \int_{0}^{1} P_{n-1}(y) P_{n}(y) w(y) \frac{d y}{1-y}
\end{aligned}
$$

In view of the compatibility conditions $\left(S_{1}\right),\left(S_{2}\right)$ and $\left(S_{2}^{\prime}\right)$, one can derive the following relations among the four auxiliary quantities $R_{n}, R_{n}^{*}, r_{n}, r_{n}^{*}$.

Proposition 3.1. From $\left(S_{1}\right)$, we obtain the following equations

$$
\begin{aligned}
r_{n+1}^{*}+r_{n}^{*} & =\alpha-\alpha_{n} R_{n}^{*}, \\
r_{n+1}+r_{n} & =\left(1-\alpha_{n}\right) R_{n}-\beta, \\
t R_{n}^{*}-(t-1) R_{n} & =2 n+1+\alpha+\beta+\gamma,
\end{aligned}
$$

where the constants $R_{n}, R_{n}^{*}, r_{n}$ and $r_{n}^{*}$ are defined in (3.6)-(3.9), respectively.

Proof. Substituting (3.4) and (3.5) into $\left(S_{1}\right)$, we have

$$
\begin{aligned}
& B_{n+1}(z)+B_{n}(z) \\
& =\frac{r_{n+1}^{*}+r_{n}^{*}}{z}-\frac{r_{n+1}+r_{n}}{z-1}+\frac{r_{n+1}+r_{n}-r_{n+1}^{*}-r_{n}^{*}-2 n-1}{z-t}
\end{aligned}
$$

and

$$
\begin{aligned}
& \left(z-\alpha_{n}\right) A_{n}(z)-\mathrm{v}^{\prime}(z) \\
& =\left(z-\alpha_{n}\right)\left[\frac{R_{n}^{*}}{z}-\frac{R_{n}}{z-1}+\frac{R_{n}-R_{n}^{*}}{z-t}\right]+\frac{\alpha}{z}+\frac{\beta}{z-1}+\frac{\gamma}{z-t} \\
& =\frac{\alpha-\alpha_{n} R_{n}^{*}}{z}-\frac{\left(1-\alpha_{n}\right) R_{n}-\beta}{z-1}+\frac{\left(t-\alpha_{n}\right)\left(R_{n}-R_{n}^{*}\right)+\gamma}{z-t} .
\end{aligned}
$$


Comparing the coefficients at $O\left(z^{-1}\right), O\left((z-1)^{-1}\right)$ and $O\left((z-t)^{-1}\right)$ in the above two formulas, we get

$$
\begin{aligned}
r_{n+1}^{*}+r_{n}^{*} & =\alpha-\alpha_{n} R_{n}^{*}, \\
r_{n+1}+r_{n} & =\left(1-\alpha_{n}\right) R_{n}-\beta, \\
r_{n+1}+r_{n}-r_{n+1}^{*}-r_{n}^{*}-2 n-1 & =\left(t-\alpha_{n}\right)\left(R_{n}-R_{n}^{*}\right)+\gamma .
\end{aligned}
$$

A combination of the above three formulas gives our proposition.

Proposition 3.2. From $\left(S_{2}^{\prime}\right)$, we have the following equations

$$
\begin{gathered}
\left(r_{n}^{*}\right)^{2}-\alpha r_{n}^{*}=\beta_{n} R_{n}^{*} R_{n-1}^{*}, \\
r_{n}^{2}+\beta r_{n}=\beta_{n} R_{n} R_{n-1} \\
(2 n+\beta+\gamma) r_{n}-(2 n+\alpha+\gamma) r_{n}^{*}+2 r_{n} r_{n}^{*}-n(n+\gamma)=\beta_{n}\left(R_{n-1} R_{n}^{*}+R_{n} R_{n-1}^{*}\right)
\end{gathered}
$$

and

$$
\begin{aligned}
\sum_{j=0}^{n-1} R_{j}= & (2 n+\alpha+\beta+\gamma)\left(r_{n}-r_{n}^{*}\right)-n(n+\gamma) \\
& +\frac{(2 n+\alpha+\beta+\gamma) r_{n}^{*}+n(n+\beta+\gamma)}{1-t}
\end{aligned}
$$

where the constants $R_{n}, R_{n}^{*}, r_{n}$ and $r_{n}^{*}$ are defined in (3.6)-(3.9), respectively.

Proof. Again we substitute (3.4) and (3.5) into $\left(S_{2}^{\prime}\right)$ to obtain

$$
\begin{aligned}
B_{n}^{2}(z)+ & \mathrm{v}^{\prime}(z) B_{n}(z)+\sum_{j=0}^{n-1} A_{j}(z) \\
= & \frac{\left(r_{n}^{*}\right)^{2}-\alpha r_{n}^{*}}{z^{2}}+\frac{r_{n}^{2}+\beta r_{n}}{(z-1)^{2}}+\frac{\left(r_{n}-r_{n}^{*}-n\right)\left(r_{n}-r_{n}^{*}-n-\gamma\right)}{(z-t)^{2}} \\
& +\frac{\alpha r_{n}-\beta r_{n}^{*}-2 r_{n} r_{n}^{*}}{z(z-1)}+\frac{\left(r_{n}-r_{n}^{*}-n\right)\left(2 r_{n}^{*}-\alpha\right)-\gamma r_{n}^{*}}{z(z-t)} \\
& -\frac{\left(r_{n}-r_{n}^{*}-n\right)\left(2 r_{n}+\beta\right)-\gamma r_{n}}{(z-1)(z-t)}+\sum_{j=0}^{n-1}\left[\frac{R_{j}^{*}}{z}-\frac{R_{j}}{z-1}+\frac{R_{j}-R_{j}^{*}}{z-t}\right]
\end{aligned}
$$


and

$$
\begin{aligned}
& \beta_{n} A_{n}(z) A_{n-1}(z) \\
& =\frac{\beta_{n} R_{n}^{*} R_{n-1}^{*}}{z^{2}}+\frac{\beta_{n} R_{n} R_{n-1}}{(z-1)^{2}}+\frac{\beta_{n}\left(R_{n}-R_{n}^{*}\right)\left(R_{n-1}-R_{n-1}^{*}\right)}{(z-t)^{2}} \\
& \quad-\frac{\beta_{n}\left(R_{n}^{*} R_{n-1}+R_{n} R_{n-1}^{*}\right)}{z(z-1)}+\frac{\beta_{n}\left(R_{n}^{*} R_{n-1}-2 R_{n}^{*} R_{n-1}^{*}+R_{n} R_{n-1}^{*}\right)}{z(z-t)} \\
& \quad+\frac{\beta_{n}\left(R_{n} R_{n-1}^{*}-2 R_{n} R_{n-1}+R_{n-1} R_{n}^{*}\right)}{(z-1)(z-t)} .
\end{aligned}
$$

Equating the coefficients of the above two formulas at $O\left(z^{-2}\right), O\left((z-1)^{-2}\right)$ and $O\left((z-t)^{-2}\right)$, we get $(3.22),(3.23)$ and

$$
\left(r_{n}-r_{n}^{*}-n\right)\left(r_{n}-r_{n}^{*}-n-\gamma\right)=\beta_{n}\left(R_{n}-R_{n}^{*}\right)\left(R_{n-1}-R_{n-1}^{*}\right)
$$

respectively. A substitution of (3.22), (3.23) into the above formula gives us (3.24). At $O\left((z-1)^{-1}\right)$, note that

$$
\frac{1}{z}=1+O(z-1), \quad \frac{1}{z-t}=\frac{1}{1-t}+O(z-1), \quad \text { as } z \rightarrow 1 .
$$

It then follows from (3.26) and (3.27) that

$$
\begin{aligned}
& -2 r_{n} r_{n}^{*}-\beta r_{n}^{*}+\alpha r_{n}-\sum_{j=0}^{n-1} R_{j}-\frac{\left(r_{n}-r_{n}^{*}-n\right)\left(2 r_{n}+\beta\right)-\gamma r_{n}}{1-t} \\
& =-\beta_{n}\left[R_{n} R_{n-1}^{*}+R_{n-1} R_{n}^{*}+\frac{-R_{n} R_{n-1}^{*}+2 R_{n} R_{n-1}-R_{n-1} R_{n}^{*}}{1-t}\right] .
\end{aligned}
$$

Combining (3.24) and the above formula, we have

$$
\begin{aligned}
\sum_{j=0}^{n-1} R_{j}= & (2 n+\alpha+\beta+\gamma)\left(r_{n}-r_{n}^{*}\right)-n(n+\gamma) \\
& +\frac{2 \beta_{n} R_{n} R_{n-1}+(2 n+\alpha+\beta+\gamma) r_{n}^{*}+n(n+\beta+\gamma)-2 r_{n}^{2}-2 \beta r_{n}}{1-t}
\end{aligned}
$$

Eliminating $\beta_{n} R_{n} R_{n-1}$ in (3.30) with the aid of (3.23), we finally obtain (3.25).

Remark 3.1. From another condition $\left(S_{2}\right)$, we get one more equation as follows

$$
(t-1)\left(r_{n+1}-r_{n}\right)-t\left(r_{n+1}^{*}-r_{n}^{*}\right)-t+\alpha_{n}=0
$$


Rewriting the above formula yields

$$
\alpha_{n}=t\left(r_{n+1}^{*}-r_{n}^{*}\right)-(t-1)\left(r_{n+1}-r_{n}\right)+t
$$

Since it follows from (1.2) and (1.4) that

$$
\alpha_{n}=\mathrm{p}_{1}(n)-\mathrm{p}_{1}(n+1), \quad n=0,1,2, \cdots
$$

with $\mathrm{p}_{1}(0):=0$, hence, it is easily seen

$$
-\sum_{j=0}^{n-1} \alpha_{j}=\mathrm{p}_{1}(n) .
$$

Inserting (3.32) into the above formula, we obtain

$$
\mathrm{p}_{1}(n)=(t-1) r_{n}-t r_{n}^{*}-n t,
$$

where we have made use of the initial conditions $r_{0}(t)=r_{0}^{*}(t):=0$.

\subsection{The recurrence coefficients}

Not only the coefficients $A_{n}(z)$ and $B_{n}(z)$ of the ladder operators in (2.1) and (2.2), but also the recurrence coefficients $\alpha_{n}$ and $\beta_{n}$ in (1.4) can be written in terms of the auxiliary quantities $R_{n}, r_{n}$ and $r_{n}^{*}$. Here we do not need $R_{n}^{*}$ since it is related to $R_{n}$ in a simple way; see (3.16).

Lemma 3.2. The recurrence coefficients $\alpha_{n}$ and $\beta_{n}$ are expressed in terms of $R_{n}, r_{n}$ and $r_{n}^{*}$ as follows:

$$
(2 n+2+\alpha+\beta+\gamma) \alpha_{n}=2(t-1) r_{n}-2 t r_{n}^{*}+(1-t) R_{n}+(\alpha+\beta+1) t-\beta
$$

and

$$
\begin{aligned}
& (2 n-1+\alpha+\beta+\gamma)(2 n+1+\alpha+\beta+\gamma) \beta_{n} \\
& =\left[t r_{n}^{*}-(t-1) r_{n}\right]^{2}-(t-1)(2 n t+\gamma t+\beta) r_{n}+t[(t-1)(2 n+\gamma)-\alpha] r_{n}^{*} \\
& \quad+n(n+\gamma)\left(t^{2}-t\right) .
\end{aligned}
$$

Proof. We use (3.14) and (3.15) to eliminate $r_{n+1}^{*}$ and $r_{n+1}$ in (3.31) and get

$$
\left[1+t R_{n}^{*}-(t-1) R_{n}\right] \alpha_{n}=t\left(\alpha-2 r_{n}^{*}\right)-(t-1)\left(R_{n}-\beta-2 r_{n}\right)+t .
$$

Substituting (3.16) into the above formula immediately gives us (3.36). 
To derive the formula for $\beta_{n}$, we need to consider the identities in Proposition 3.2. Multiplying both sides of (3.22) by $t^{2}$ and eliminating $t^{2} R_{n}^{*} R_{n-1}^{*}$ with the aid of (3.16), we have

$$
t^{2}\left(\left(r_{n}^{*}\right)^{2}-\alpha r_{n}^{*}\right)=(t-1) \beta_{n}\left[(t-1) R_{n-1} R_{n}+c_{n-1} R_{n}+c_{n} R_{n-1}\right]+c_{n-1} c_{n} \beta_{n},
$$

where $c_{n}:=2 n+1+\alpha+\beta+\gamma$. Similarly, we multiply both sides of (3.24) by $t$ and us (3.16) again to get

$t\left[(2 n+\beta+\gamma) r_{n}-(2 n+\alpha+\gamma) r_{n}^{*}+2 r_{n} r_{n}^{*}-n(n+\gamma)\right]=\beta_{n}\left[2(t-1) R_{n-1} R_{n}+c_{n-1} R_{n}+c_{n} R_{n-1}\right]$.

On account of (3.23), it is readily derived from the above formula that

$$
\begin{aligned}
& t\left[(2 n+\beta+\gamma) r_{n}-(2 n+\alpha+\gamma) r_{n}^{*}+2 r_{n} r_{n}^{*}-n(n+\gamma)\right]-(t-1)\left(r_{n}^{2}+\beta r_{n}\right) \\
& \quad=\beta_{n}\left[(t-1) R_{n-1} R_{n}+c_{n-1} R_{n}+c_{n} R_{n-1}\right] .
\end{aligned}
$$

Substituting (3.40) into (3.39) yields (3.37).

Remark 3.2. For $n=0$, from (1.4) and the definitions of $R_{0}(t), r_{0}(t)$ and $r_{0}^{*}(t)$, it follows that

$$
\begin{aligned}
\alpha_{0}(t) & =\frac{(\alpha+1)_{2} F_{1}\left(\alpha+2,-\gamma, \alpha+\beta+3 ; \frac{1}{t}\right)}{(\alpha+\beta+2)_{2} F_{1}\left(\alpha+1,-\gamma, \alpha+\beta+2 ; \frac{1}{t}\right)}, \\
R_{0}(t) & =\frac{(\alpha+\beta+1)_{2} F_{1}\left(\alpha+1,-\gamma, \alpha+\beta+1 ; \frac{1}{t}\right)}{{ }_{2} F_{1}\left(\alpha+1,-\gamma, \alpha+\beta+2 ; \frac{1}{t}\right)}, \\
r_{0}(t) & =r_{0}^{*}(t)=0,
\end{aligned}
$$

where ${ }_{2} F_{1}$ is the hypergeometric function; see [1, p.556]. The validity of (3.36) at $n=0$ can be verified directly from the above formulas.

Furthermore, it is easily seen that

$$
R_{0}(t)=\alpha+\beta+1+O(1 / t)
$$

as $t \rightarrow-\infty$.

\subsection{The $t$ dependance}

Recall that our weight function depends on $t$, therefore, all of the quantities considered in this paper such as the coefficient of generalized Jacobi polynomials, Hankel determinant, etc., can be viewed as functions in $t$. In this subsection, we will investigate their dependance with respect to this parameter. We start with the study the coefficient $\mathrm{p}_{1}(n)$ in (1.2). 
Lemma 3.3. We have

$$
\frac{d}{d t} p_{1}(n)=r_{n}-r_{n}^{*}-n
$$

Proof. From the orthogonal property (1.1), we know

$$
\int_{0}^{1} P_{n}(x) P_{n-1}(x) w(x ; t) d x=0 .
$$

Note that $P_{n}(x)$ is also dependent on $t$. Taking derivative of the above formula with respect to $t$ gives us

$$
\int_{0}^{1} \frac{d}{d t} P_{n}(x) P_{n-1}(x) w(x ; t) d x+\int_{0}^{1} P_{n}(x) P_{n-1}(x) \frac{d}{d t} w(x ; t) d x=0 .
$$

It then follows from (1.1) $-(1.3)$ that

$$
h_{n-1} \frac{d}{d t} \mathrm{p}_{1}(n)-\gamma \int_{0}^{1} P_{n}(x) P_{n-1}(x) w(x) \frac{d x}{x-t}=0 .
$$

Combining (3.8), (3.9) and (3.13), we get (3.42) immediately.

By (3.35) and the above lemma, it is easily seen that

$$
\frac{d}{d t} \mathrm{p}_{1}(n)=r_{n}-r_{n}^{*}-n=r_{n}+(t-1) \frac{d}{d t} r_{n}-r_{n}^{*}-t \frac{d}{d t} r_{n}^{*}-n .
$$

Hence, we obtain the following nice relation between the derivatives of $r_{n}$ and $r_{n}^{*}$

$$
t \frac{d}{d t} r_{n}^{*}=(t-1) \frac{d}{d t} r_{n}
$$

Next, we derive the following property about the Hankel determinant $D_{n}(t)$.

Lemma 3.4. We have

$$
t \frac{d}{d t} \ln D_{n}(t)=n(n+\alpha+\beta+\gamma)-\sum_{j=0}^{n-1} R_{j}
$$

where $R_{j}$ is defined in (3.7).

Proof. Differentiating (1.1) with respect to $t$ yields

$$
h_{n}^{\prime}=-\gamma \int_{0}^{1}\left[P_{n}(x)\right]^{2} w(x) \frac{d x}{x-t} .
$$


This, together with (3.6), (3.7) and (3.12) implies

$$
h_{n}^{\prime}=h_{n}\left(R_{n}^{*}-R_{n}\right)
$$

Using (3.16) to replace $R_{n}^{*}$ by $R_{n}$, we find

$$
t \frac{d}{d t} \ln h_{n}=2 n+1+\alpha+\beta+\gamma-R_{n} .
$$

Then, a combination of (3.48) and (1.12) gives us (3.45).

Finally, we derive differential equations for the recurrence coefficients $\alpha_{n}$ and $\beta_{n}$. They are the non-standard Toda equations.

Lemma 3.5. The recurrence coefficients $\alpha_{n}$ and $\beta_{n}$ satisfy the following differential equations

$$
\begin{gathered}
t \frac{d}{d t} \alpha_{n}=\alpha_{n}+r_{n}-r_{n+1}, \\
t \frac{d}{d t} \beta_{n}=\left(2+R_{n-1}-R_{n}\right) \beta_{n},
\end{gathered}
$$

where $R_{n}$ and $r_{n}$ are defined in (3.7) and (3.9), respectively.

Proof. Applying $t \frac{d}{d t}$ to both sides of (3.33), we have from (3.42)

$$
t \frac{d}{d t} \alpha_{n}=t\left(r_{n}-r_{n}^{*}-n\right)-t\left(r_{n+1}-r_{n+1}^{*}-n-1\right) .
$$

$\left(T_{1}\right)$ then follows from (3.31) and the above formula. Using (3.48), it is readily seen that

$$
t \frac{d}{d t} \ln \frac{h_{n}}{h_{n-1}}=2+R_{n-1}-R_{n} .
$$

Note that $\beta_{n}=h_{n} / h_{n-1}$, one easily gets $\left(T_{2}\right)$ from the above formula.

\section{Proof of Theorem 1.1}

Now we are ready to prove our main theorem. The idea is to make use of Lemma 3.4 to express $r_{n}^{*}$ and $r_{n}$ in terms of $H_{n}$ and its derivative with respect to $t$. Then we derive two independent formulas for $R_{n}$ in terms of $r_{n}^{*}$ and $r_{n}$ with the aid of (3.37) and $\left(T_{2}\right)$. Equating these two formulas, finally we obtain an equation involving $H_{n}$, $H_{n}^{\prime}$ and $H_{n}^{\prime \prime}$. We first need the following proposition for $r_{n}$ and $r_{n}^{*}$. 


\section{Proposition 4.1.}

$$
\begin{aligned}
& r_{n}^{*}=-\frac{n(n+\beta+\gamma)+(t-1) H_{n}^{\prime}-H_{n}}{2 n+\alpha+\beta+\gamma} \\
& r_{n}=\frac{n(n+\alpha+\gamma)-t H_{n}^{\prime}+H_{n}}{2 n+\alpha+\beta+\gamma}
\end{aligned}
$$

Proof. Recalling the definition of $H_{n}$ in (1.7), we substitute (3.25) into (3.45) and get

$$
\begin{aligned}
H_{n}= & {\left[n(2 n+\alpha+\beta+2 \gamma)-(2 n+\alpha+\beta+\gamma)\left(r_{n}-r_{n}^{*}\right)\right](t-1) } \\
& +(2 n+\alpha+\beta+\gamma) r_{n}^{*}+n(n+\beta+\gamma),
\end{aligned}
$$

Taking a derivative of the above formula with respect to $t$, it then follows from (3.44) that

$$
(t-1) H_{n}^{\prime}-H_{n}=-n(n+\beta+\gamma)-(2 n+\alpha+\beta+\gamma) r_{n}^{*}
$$

which gives us (4.1). Eliminating $r_{n}^{*}$ from the above two formulas, we get (4.2).

Next we derive a proposition for $R_{n}$ as follows.

Proposition 4.2. The auxiliary quantity $R_{n}$ has the following representations

$$
\begin{aligned}
R_{n}(t) & =\frac{(2 n+1+\alpha+\beta+\gamma)\left[l\left(r_{n}, r_{n}^{*}, t\right)-t(1-t) r_{n}^{\prime}(t)\right]}{2 k\left(r_{n}, r_{n}^{*}, t\right)} \\
\frac{1}{R_{n}(t)} & =\frac{l\left(r_{n}, r_{n}^{*}, t\right)+t(1-t) r_{n}^{\prime}(t)}{2(2 n+1+\alpha+\beta+\gamma)\left(\beta+r_{n}\right) r_{n}}
\end{aligned}
$$

where

$$
l\left(r_{n}, r_{n}^{*}, t\right):=2(1-t) r_{n}^{2}+\left[(2 n-\beta+\gamma) t+2 \beta+2 t r_{n}^{*}\right] r_{n}-(2 n+\alpha+\gamma) t r_{n}^{*}-n(n+\gamma) t
$$

and

$$
\begin{aligned}
k\left(r_{n}, r_{n}^{*}, t\right):= & {\left[r_{n}^{*}-(t-1) r_{n}\right]^{2}-(t-1)(2 n t+\gamma t+\beta) r_{n} } \\
& +t[(t-1)(2 n+\gamma)-\alpha] r_{n}^{*}+n(n+\gamma)\left(t^{2}-t\right) .
\end{aligned}
$$

Proof. Using (3.23), we eliminate $R_{n-1}$ in (3.40) and obtain

$$
\begin{aligned}
& (2 n+1+\alpha+\beta+\gamma) \frac{r_{n}^{2}+\beta r_{n}}{R_{n}}+(2 n-1+\alpha+\beta+\gamma) \beta_{n} R_{n} \\
& =t\left[(2 n+\beta+\gamma) r_{n}-(2 n+\alpha+\gamma) r_{n}^{*}+2 r_{n} r_{n}^{*}-n(n+\gamma)\right]-2(t-1)\left(r_{n}^{2}+\beta r_{n}\right) .
\end{aligned}
$$


Replacing $\beta_{n}$ in the above formula with the aid of (3.37), we have

$$
\begin{aligned}
& \frac{2 n+1+\alpha+\beta+\gamma}{R_{n}}\left(r_{n}^{2}+\beta r_{n}\right)+\frac{k\left(r_{n}, r_{n}^{*}, t\right)}{2 n+1+\alpha+\beta+\gamma} R_{n} \\
& =2(1-t) r_{n}^{2}+\left[(2 n-\beta+\gamma) t+2 \beta+2 r_{n}^{*} t\right] r_{n}-(2 n+\alpha+\gamma) t r_{n}^{*}-n(n+\gamma) t
\end{aligned}
$$

On the other hand, by applying $t \frac{d}{d t}$ to (3.37), it follows

$$
\begin{aligned}
& (2 n-1+\alpha+\beta+\gamma)(2 n+1+\alpha+\beta+\gamma) t \frac{d}{d t} \beta_{n} \\
& =t\left[2 t\left(r_{n}^{*}\right)^{2}+2(1-2 t) r_{n} r_{n}^{*}+2(t-1) r_{n}^{2}+(2 n-\beta+\gamma-(4 n+2 \gamma) t) r_{n}\right. \\
& \quad-(2 n+\alpha+\gamma-(4 n+2 \gamma) t) r_{n}^{*}-(t-1)(2 n t+\gamma t+\beta) \frac{d}{d t} r_{n} \\
& \left.\quad+t((t-1)(2 n+\gamma)-\alpha) \frac{d}{d t} r_{n}^{*}+n(n+\gamma)(2 t-1)\right],
\end{aligned}
$$

where we have used (3.44). A further substitution of (3.23), (3.37) and $\left(T_{2}\right)$ into the above formula yields

$$
\begin{gathered}
\frac{(2 n-1+\alpha+\beta+\gamma)(2 n+1+\alpha+\beta+\gamma)}{R_{n}}\left(r_{n}^{2}+\beta r_{n}\right)-k\left(r_{n}, r_{n}^{*}, t\right) R_{n} \\
=2(t-1) r_{n}^{2}-\left[(2 n-\beta+\gamma) t+2 \beta+2 r_{n}^{*} t\right] r_{n}+(2 n+\alpha+\gamma) t r_{n}^{*} \\
+n(n+\gamma) t+t(1-t)(2 n+\alpha+\beta+\gamma) \frac{d}{d t} r_{n} .
\end{gathered}
$$

Formulas (4.5) and (4.6) now follow from solving for $R_{n}$ and $1 / R_{n}$ from (4.9) and (4.10).

Now we are ready to finish the proof of Theorem 1.1. Proof of Theorem 1.1. Multiplying (4.5) and (4.6) gives us

$$
t^{2}(t-1)^{2}\left[r_{n}^{\prime}(t)\right]^{2}=l^{2}\left(r_{n}, r_{n}^{*}, t\right)-4 k\left(r_{n}, r_{n}^{*}, t\right)\left(\beta+r_{n}\right) r_{n},
$$

where $l\left(r_{n}, r_{n}^{*}, t\right)$ and $k\left(r_{n}, r_{n}^{*}, t\right)$ are given in (4.7) and (4.8), respectively. Recall that $r_{n}^{*}$ and $r_{n}$ can be written in terms of $H_{n}$ and $H_{n}^{\prime}$, see (4.1) and (4.2). Therefore, the above formula actually gives us a non-linear differential equation for $H_{n}$. Using (1.8) to replace $H_{n}$ by $\widetilde{H}_{n}$, we finally get (1.9), which completes the proof of our theorem. 


\section{Proof of Theorem 1.2}

We conclude this paper with the proof of Theorem 1.2 .

Proof of Theorem 1.2. Firstly, we try to express $r_{n}^{*}$ in terms of $r_{n}, r_{n}^{\prime}, R_{n}$ and $R_{n}^{\prime}$. To achieve this, we substitute (3.36) into $\left(T_{1}\right)$ and get an equation involving $r_{n}, r_{n}^{\prime}, r_{n}^{*}$, $r_{n}^{* \prime}, R_{n}^{\prime}$ and $r_{n+1}$. Then we use (3.15) and (3.44) to eliminate $r_{n+1}$ and $r_{n}^{* \prime}$. At the end, we arrive at

$$
\begin{aligned}
r_{n}^{*}= & \frac{1}{2}+\frac{1}{2 R_{n}}\left((t-1) R_{n}^{\prime}-2 r_{n}-(\alpha+\beta+1)\right)+\frac{1}{2 t R_{n}}((2 n+\alpha+\beta+\gamma+2) \\
& \left.\times\left(2 r_{n}-R_{n}+\beta\right)+\left(R_{n}+1\right)\left[2(t-1) r_{n}-(t-1) R_{n}+(\alpha+\beta+1) t-\beta\right]\right)
\end{aligned}
$$

Next, we insert the above formula into (4.5) and (4.6) and obtain a pair of linear equations in $r_{n}$ and $r_{n}^{\prime}$. Solving this linear system gives us

$$
r_{n}=F\left(R_{n}, R_{n}^{\prime}\right) \quad \text { and } \quad r_{n}^{\prime}=G\left(R_{n}, R_{n}^{\prime}\right),
$$

where $F(\cdot, \cdot)$ and $G(\cdot, \cdot)$ are functions that can be explicitly computed. Because the expressions are too complicated, we have decided not to write them down. Due to the fact that $\frac{d}{d t} F\left(R_{n}, R_{n}^{\prime}\right)=r_{n}^{\prime}=G\left(R_{n}, R_{n}^{\prime}\right)$, it can be shown that

$$
\begin{aligned}
& {[(2 n+\alpha+\beta+\gamma)(2 n+\alpha+\beta+\gamma+1)+((2 n+\alpha+\beta+\gamma+1) t-2(2 n+\alpha+\beta+\gamma)} \\
& \left.-1) R_{n}(t)-(t-1) R_{n}^{2}(t)+t(t-1) R_{n}^{\prime}(t)\right] \Phi\left(R_{n}, R_{n}^{\prime}, R_{n}^{\prime \prime}\right)=0
\end{aligned}
$$

where $\Phi(\cdot, \cdot, \cdot)$ is a functions that is explicitly known. Obviously, the above formula yields two differential equations. One is a first order differential equation, actually a Riccati equation, whose solution is given by

$$
R_{n}(t)=\frac{(2 n+\alpha+\beta+\gamma+1)\left(1+\lambda(2 n+\alpha+\beta+\gamma+1)(1-t)^{2 n+\alpha+\beta+\gamma}\right)}{1+\lambda(2 n+\alpha+\beta+\gamma+1)(1-t)^{2 n+\alpha+\beta+\gamma+1}},
$$

where $\lambda$ is an integration constant. However, as $t \rightarrow-\infty$, it is easily seen that

$$
R_{n}(t) \rightarrow \begin{cases}0, & \text { if } \lambda \neq 0 \\ 2 n+\alpha+\beta+\gamma+1, & \text { if } \lambda=0\end{cases}
$$

which violates the result $R_{0}(t) \sim \alpha+\beta+1$ in (3.41). So we discard this Riccati equation. 
Finally, applying a suitable rescaling and displacement as given in (1.15), we obtain the Painlevé VI equation (1.16) from $\Phi(\cdot, \cdot, \cdot)=0$ in (5.3). And this finishes the proof of our theorem.

\section{Acknowledgement}

We thank the referees for providing constructive comments to improve the contents of this paper.

\section{References}

[1] M. Abramowitz and I.A. Stegun, Handbook of Mathematical Functions with Formulas, Graphs, and Mathematical Tables, reprint of the 1972 edition, Dover Publications, New York, 1992.

[2] E. Basor and Y. Chen, Painlevé V and the distribution function of a discontinuous linear statistics in the Laguerre unitary ensembles, J. Phys. A.: Math. Theor., 42 (2009) 035203 (18pp).

[3] E. Basor, Y. Chen and T. Ehrhardt, Painlevé V and time dependent Jacobi polynomials, arXiv:0905.2620. To appear in J. Phys. A.

[4] S. Bonan and D.S. Clark, Estimates of the orthogonal polynomials with weight $\exp \left(-x^{m}\right), m$ an even positive integer, J. Approx. Theory, 46 (1986), 408-410.

[5] S. Bonan, D.S. Lubinsky and P. Nevai, Orthogonal polynomials and their derivatives, II, SIAM J. Math. Anal., 18 (1987), 1163-1176.

[6] S. Bonan and P. Nevai, Orthogonal polynomials and their derivatives, I, J. Approx. Theory, 40 (1984), 134-147.

[7] A. Borodin and P. Deift, Fredholm determinants, Jimbo-Miwa-Ueno $\tau$-functions, and representation theory, Comm. Pure Appl. Math., 55 (2002), no. 9, 11601230.

[8] Y. Chen and D. Dai, Painlevé V and a Pollaczek-Jacobi type orthogonal polynomials, arXiv:0809.3641.

[9] Y. Chen and M. V. Feigin, Painlevé IV and degenerate Gaussian unitary ensembles, J. Phys. A, 39 (2006), 12381-12393. 
[10] Y. Chen and M. Ismail, Ladder operators and differential equations for orthogonal polynomials, J. Phys. A, 30 (1997), no. 22, 7817-7829.

[11] Y. Chen and M. Ismail, Jacobi polynomials from compatibility conditions, Proc. Amer. Math. Soc., 133 (2005), no. 2, 465-472.

[12] Y. Chen, and A.R. Its, Painlevé III and a singular linear statistics in Hermitian random matrix ensembles, I, J. Approx. Theory (2009), doi:10.1016/j.jat.2009.05.005.

[13] P.J. Forrester and N.S. Witte, Random matrix theory and the sixth Painlevé equation, J. Phys. A, 39 (2006), no. 39, 12211-12233.

[14] A. Foulquie Moreno, A. Martinez-Finkelshtein and V.L. Sousa, On a conjecture of A. Magnus concerning the asymptotic behavior of the recurrence coefficients of the generalized Jacobi polynomials, arXiv:0905.2753.

[15] J.L. Gammel, and J. Nuttall, Note on generalized Jacobi polynomials, The Riemann problem, complete integrability and arithmetic applications (Buressur-Yvette/New York, 1979/1980), pp. 258-270, Lecture Notes in Math., 925, Springer, Berlin-New York, 1982.

[16] L. Haine and J. Semengue, The Jacobi polynomial ensemble and the Painlevé VI equation, J. Math. Phys., 40 (1999), no. 4, 2117-2134.

[17] M.E.H. Ismail, Classical and Quantum Orthogonal Polynomials in One Variable, Encyclopedia of Mathematics and its Applications, 98., Cambridge University Press, Cambridge, 2005.

[18] M. Jimbo and T. Miwa, Monodromy perserving deformation of linear ordinary differential equations with rational coefficients, II, Physica D, 2 (1981), 407-448.

[19] A.P. Magnus, Painlevé-type differential equations for the recurrence coefficients of semi-classical orthogonal polynomials, J. Comput. Appl. Math., 57 (1995), no. 1-2, 215-237.

[20] M. L. Mehta, Random Matrices, Third Ed. Elsevier, New York, 2004.

[21] P. Nevai, T. Erdélyi and A.P. Magnus, Generalized Jacobi weights, Christoffel functions, and Jacobi polynomials, SIAM J. Math. Anal., 25 (1994), no. 2, 602614. 
[22] K. Okamoto, On the $\tau$-function of the Painlevé equations, Physica D, 2 (1981), $525-535$.

[23] J. Shohat, A differential equation for orthogonal polynomials, Duke Math. J., 5 (1939), 401-417.

[24] G. Szegö, Orthogonal Polynomials, 4th edition, AMS Colloquium Publications, Vol. 23, Amer. Math. Soc., Providence R.I., 1975.

[25] M. Vanlessen, Strong asymptotics of the recurrence coefficients of orthogonal polynomials associated to the generalized Jacobi weight, J. Approx. Theory, 125 (2003), no. 2, 198-237.

[26] P. Vértesi, Uniform asymptotics of derivatives of orthogonal polynomials based on generalized Jacobi weights, Acta Math. Hungar., 85 (1999), no. 1-2, 97-130. 